\title{
Young's Modulus Measurement of Submicron-thick Aluminum Films Using Fan-shaped Silicon Resonators
}

\author{
Takahiro Namazu, ${ }^{1 *}$ Junki Kuroishi, ${ }^{2}$ Hiroya Yamagiwa, ${ }^{2}$ Daisuke Goto, ${ }^{2}$ \\ Tatsuya Takeuchi, ${ }^{3}$ Kohei Murakami, ${ }^{3}$ Yasushi Kawashimo, ${ }^{3}$ and Tetsuo Takano ${ }^{4}$ \\ ${ }^{1}$ Department of Mechanical Engineering, Aichi Institute of Technology, \\ 1247 Yachigusa, Yakusa, Toyota, Aichi 470-0392, Japan \\ ${ }^{2}$ Department of Mechanical Engineering, University of Hyogo, \\ 2167 Shosha, Himeji, Hyogo 671-2201, Japan \\ ${ }^{3}$ Sinko Seiki Co., Ltd., 1-35-3 Takatsukadai, Nishi, Kobe, Hyogo 651-2271, Japan \\ ${ }^{4}$ The New Industry Research Organization, \\ 1-5-2 Minatojima Minamimachi, Chuo, Kobe, Hyogo 650-0047, Japan
}

(Received July 22, 2018; accepted November 1, 2018)

Keywords: submicron-thick Al films, vacuum evaporation, MEMS resonator, resonant frequency, Young's modulus, material property measurement

In this paper, we describe a way of finding the optimum resonator geometry required to determine a reasonably accurate Young's modulus of submicron-thick Al films. The films with thicknesses ranging from 15 to $380 \mathrm{~nm}$ are deposited onto the back of specially designed fanshaped resonators by vacuum evaporation. Young's modulus is calculated from the difference in resonant frequencies obtained before and after the deposition. By using resonators with the support beam length larger than $75 \mu \mathrm{m}$ and cross-sectional aspect ratio larger than 1.0, the measured Young's moduli of the Al films are close to the bulk value. When the films were thicker than $50 \mathrm{~nm}$, the moduli show no film thickness effect (57.5 $\pm 5.8 \mathrm{GPa}$ on average). Young's moduli measured by resonance testing are compared with those measured by nanoindentation testing. The reliability of the measured Young's moduli is discussed in light of resonant frequency and film thickness measurement cancellations of significant digits.

\section{Introduction}

Submicron-thick films made of a variety of materials, such as metals, alloys, polymers, and ceramics, are commonly used in semiconductor devices and micro/nano-electromechanical systems (MEMS/NEMS). ${ }^{(1)}$ Almost all of the films play some kind of functional roles, for example, surface passivation, diffusion barrier, wiring, and base structure. One important thing impossible to overlook is that these functional films are part of the structural components of a device. In semiconductor devices and MEMS/NEMS, submicron-thick films are subjected to thermal stress and/or mechanical stress, which will degrade the performance and reliability of these devices. For the achievement of safe and reliable devices with steady performance and long-term durability, understanding of the mechanical properties of films in addition to their functional performances through experiments is required. ${ }^{(2)}$

*Corresponding author: e-mail: tnamazu@aitech.ac.jp

https://doi.org/10.18494/SAM.2019.2064 
Knowledge of the mechanical properties of submicron-thick films is indispensable for the structural design of devices with such thin films. To experimentally measure the mechanical characteristics at the nano- or submicron scale, tensile, ${ }^{(3-11)}$ bulge,${ }^{(12,13)}$ ultrasonic, ${ }^{(14-16)}$ and nanoindentation $^{(17,18)}$ tests are typically performed. Among these tests, the tensile test is representative even for nanomaterials, although is well known as a commonly used method for bulk materials. Its strongest point is the direct obtainment of the stress-strain relation, which can provide various fundamental mechanical properties, such as Young's modulus, yield strength, work-hardening coefficient, tensile strength, fracture strength, and breaking strain. If lateral strain can be measured experimentally, Poisson's ratio can also be determined. However, the experimental setup, which includes nanoscale specimen preparation, specimen chucking, and force and displacement measurements, is at a very high level; therefore, it is very difficult to precisely perform the test. The bulge test is a method that has been developed only for thin films. The test is technically advantageous to membrane sample preparation and force application, which means the test is simple. However, nonuniform stress distribution, which leads to deviation from the true value, around the membrane-substrate boundary is sure to occur, which might reduce the accuracy of the obtained mechanical characteristic values. Scanning acoustic microscopy has potential as a nondestructive method for directly measuring Young's modulus of submicron-thick films. The acoustic method is superior in that specimen preparation, where a film is merely deposited onto a substrate, is easy, so it is suitable particularly for hard coating films, such as diamond-like carbon. ${ }^{(14)}$ However, for accurate measurement in submicron-thick film specimens, the method requires a wide in-plane area and a constant film thickness. The nanoindentation test is a powerful tool for obtaining the mechanical characteristics of film materials, but advantages and disadvantages coexist in the method. The effect of the substrate is critical in discussing the true mechanical properties of films. When the penetration depth is larger than the film thickness, the obtained results are sure to include not only the film's mechanical properties but also the substrate's properties. Although it is typically known that the depth should be controlled to be less than one-tenth the film thickness, this control with submicron-thick films is quite difficult. Another difficulty is the effect of indenter tip shape. When the depth is smaller than the film thickness (although the depth is always small for submicron-thick films), a high roundness of the indenter definitely yields a negative effect on the accurate derivation of the mechanical properties. Therefore, even though these experimental techniques, for which commercial test equipment is already available, are used, technical difficulties in accurately measuring the mechanical properties of submicron-thick films still remain.

The purpose of this work is to establish an experimental technique using the designed and produced MEMS resonators made of single-crystal Si (SCS) for quantitatively and simply measuring Young's moduli of submicron-thick films. In this work, we prepare 15-380-nmthick Al films deposited by vacuum evaporation, and experimentally measure their Young's moduli by the resonance test method. ${ }^{(19-21)}$ The reliability of the measured values is discussed from the viewpoint of the cancellation of significant digits of measured resonant frequency and film thickness values. Also, the Young's moduli are compared with those obtained by the nanoindentation test, and the usefulness of the resonance method is discussed. 


\section{Derivation of Young's Modulus}

Figure 1 shows a photograph of the designed and produced MEMS resonator along with its schematic. The resonator consists of a fan-shaped vibration body, a combdrive electrostatic actuator for resonance motion generation, a capacitive sensor for vibration amplitude measurement, a gauge for in-plane rotation angle measurement using a charge-coupled device (CCD) image analysis system, and a rectangular solid beam for rotation support. The basic design was proposed by Muhlstein and co-workers. ${ }^{(22-30)}$ Using the device, they investigated fatigue phenomena in poly-crystal $\mathrm{Si}^{(22-27)} \mathrm{SCS}^{(28,29)}$ and other materials. ${ }^{(30)}$ We redesigned their resonator to optimize the shape and dimensions for the quantitative measurement of Young's moduli of submicron-thick films. The redesign of the resonator was conducted in consideration of two things: matching the deformation of the film and substrate, and matching the shape and dimensions of the actual device to Eqs. (1)-(3) below. The redesigned resonators were fabricated from 4 in. silicon-on-insulator (SOI) wafers with a 5 - $\mu \mathrm{m}$-thick device layer and a $0.2-\mu \mathrm{m}$-thick buried-oxide layer by conventional micromachining technologies including deep reactive ion etching (DRIE). Since the smooth sidewalls of a resonator are important for the accurate derivation of Young's modulus, focused ion beam (FIB) milling for removing a scallop pattern on the sidewalls was performed. For the support beam in the resonators used in this work, the length was $25-100 \mu \mathrm{m}$ and the cross-sectional aspect ratio was $0.4-2.3$. Resonators with a longer support beam failed during the fabrication process; thus, unfortunately, they could not be used in this work.

On the basis of the linear elastic theory for isotropic materials, the resonant frequency $f$ for this type of resonator before film deposition is given as ${ }^{(31)}$

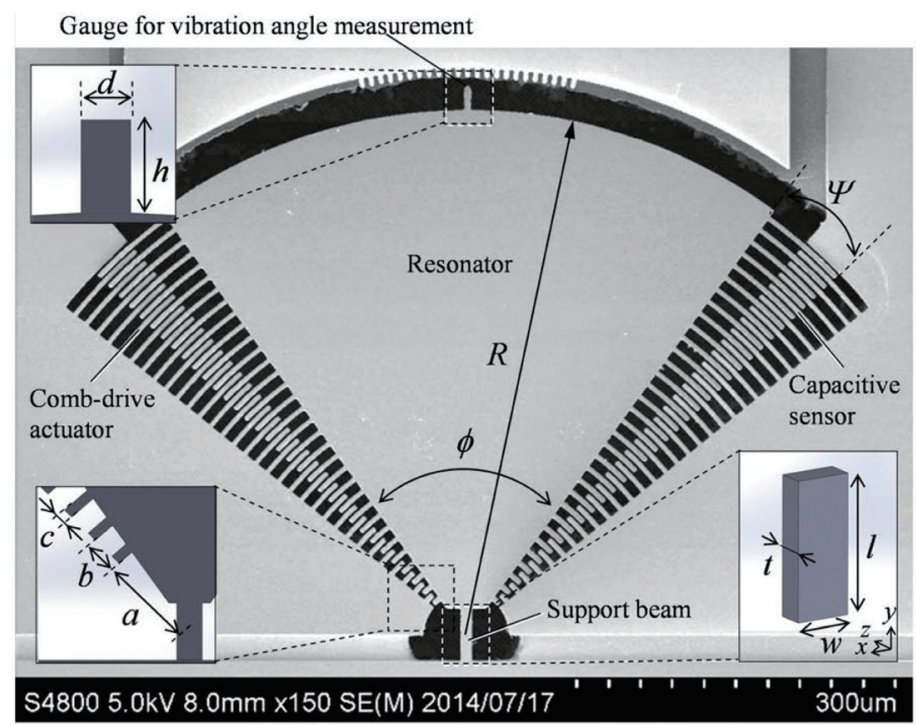

Fig. 1. Photograph of fan-shaped in-plane-motion MEMS resonator fabricated by conventional micromachining technologies. The dimensions of all the mechanical parts were used for the calculation of Young's moduli of the Al film and SCS substrate. 


$$
f=\frac{1}{2 \pi} \sqrt{\frac{k}{J}}=\frac{1}{2 \pi} \sqrt{\frac{E w^{3}}{3 \rho l X}}
$$

where $k$ and $J$ are the spring constant of the rotation support beam and the moment of inertia of the resonator, respectively, which can be expressed as

$$
k=\frac{E t w^{3}}{12 l}
$$

and

$$
J=\frac{1}{4} \rho t\left[\varphi R^{4}+2 \psi \sum_{n=0}^{m-1}\left\{(a+b n+c)^{4}-(a+b n)^{4}\right\}+d h\left\{\frac{d^{2}+h^{2}}{3}+(2 R+h)^{2}\right\}\right] .
$$

$E$ and $\rho$ are Young's modulus and density of the structural material of the resonator, respectively. ${ }^{(31)}$ $X$ in Eq. (1) indicates the terms inside the large square bracket in Eq. (3). Other dimensional parameters in Eqs. (1)-(3) are defined in Fig. 1. Equation (1) indicates that, by experimentally measuring $f, E$ can be obtained directly.

After $f$ was measured in the experiment, Al film was deposited by vacuum evaporation. Since the top surface of the device chip includes wirings for voltage-signal application and electric charge measurement, the film was deposited onto the back of the resonators made with a device layer of a SOI wafer, as illustrated in Fig. 2. A 1.0-mm-diameter Al wire with a purity of $99.999 \%$ was used as a target material. A current of 50 A was applied to a tungsten boat for Al evaporation. The film thickness was controlled by changing the evaporation time. Since there is a through-hole beneath each resonator fabricated by DRIE, the film can be deposited onto only the resonator's back surface (without deposition onto the top surface). In addition, the deposition was conducted from directly underneath the through-hole to deposit the Al film as uniform as possible onto a resonator. If the deposition is conducted at an inclined angle, owing to the shadowing effect of the wall of the handling wafer around the through-hole, the deposited film thickness is not uniform. With this deposition, the resonators become double layered: an Al film layer plus a SCS layer. Only the thickness of the resonators is changed (no other inplane dimensions are changed).

As the result, the resonant frequency $f_{1+2}$ of double-layered resonators after the deposition is changed from the $f$ of single-layer resonators and can be expressed as

$$
f_{1+2}=\frac{1}{2 \pi} \sqrt{\frac{E_{1+2} w^{3}}{3 \rho_{1+2} l X}}
$$

where the suffixes 1 and 2 are indicative of the SCS substrate and Al film, respectively. $E_{1+2}$ 


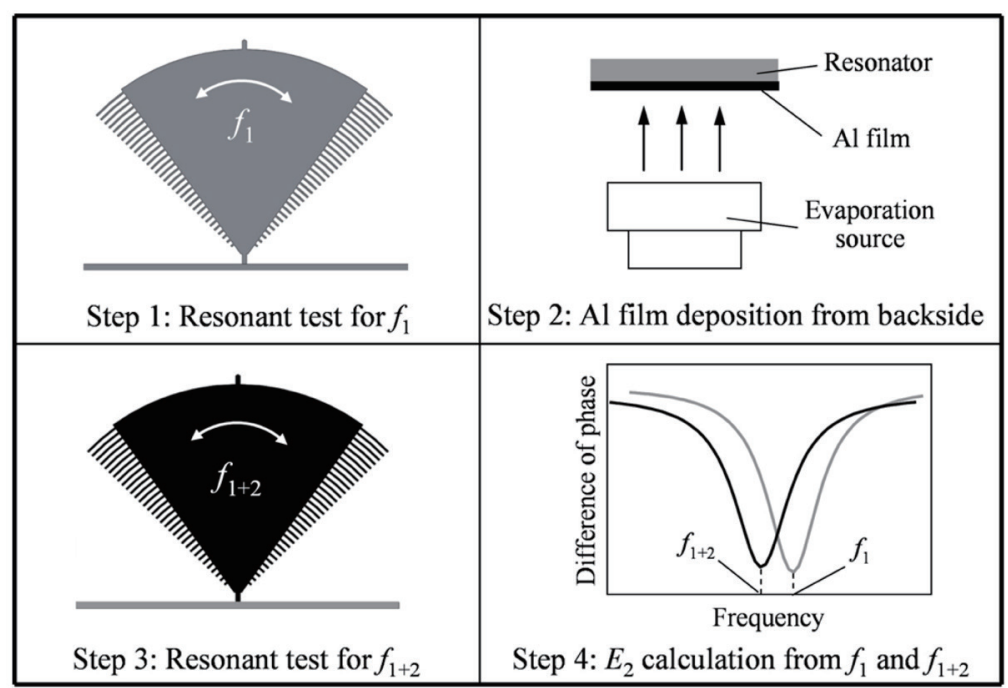

Fig. 2. Schematic of Young's modulus derivation for Al film. First, the resonant frequency $f_{1}$ for the SCS resonator is measured. Then, an $\mathrm{Al}$ film is deposited onto the back by vacuum evaporation. After that, the resonant frequency $f_{1+2}$ for the Al-film-deposited SCS resonator is measured. Young's modulus of the Al film is determined from the difference in resonant frequency between before and after the film deposition.

is the apparent Young's modulus of the support beam consisting of double layers with the SCS substrate and $\mathrm{Al}$ film. Assuming that all the materials used obey the linear elastic theory, $E_{1+2}$ is given as ${ }^{(32)}$

$$
E_{1+2}=\frac{t_{1}}{t_{1}+t_{2}} \cdot \frac{E_{1}}{1-v_{1}^{2}}+\frac{t_{2}}{t_{1}+t_{2}} \cdot \frac{E_{2}}{1-v_{2}^{2}}-\frac{\left(\frac{t_{1}}{t_{1}+t_{2}} \cdot \frac{E_{1} v_{1}}{1-v_{1}^{2}}+\frac{t_{2}}{t_{1}+t_{2}} \cdot \frac{E_{2} v_{2}}{1-v_{2}^{2}}\right)^{2}}{\frac{t_{1}}{t_{1}+t_{2}} \cdot \frac{E_{1}}{1-v_{1}^{2}}+\frac{t_{2}}{t_{1}+t_{2}} \cdot \frac{E_{2}}{1-v_{2}^{2}}}
$$

where $E, v$, and $t$ are Young's modulus, Poisson's ratio, and thickness, respectively. The subscripts 1 and 2 are indicative of the SCS substrate and Al film, respectively. When $v_{1}$ is identical to $v_{2}, E_{1+2}$ can be expressed as

$$
E_{1+2}=\frac{E_{1} t_{1}+E_{2} t_{2}}{t_{1}+t_{2}}
$$

The calculation of Eq. (5) including high-order terms is very complicated, even if $v_{2}$ is known prior to the experiment. By contrast, Eq. (6) can be roughly and simply calculated, although it may have an uncertainty of several percent at most. ${ }^{(33)}$ The uncertainty is quantitatively discussed later. For simple calculation, we have decided to use Eq. (6) in this study.

The mean density $\rho_{1+2}$ of the double-layered resonators in Eq. (4) can be expressed as 


$$
\rho_{1+2}=\frac{\rho_{1} t_{1}+\rho_{2} t_{2}}{t_{1}+t_{2}}
$$

where $\rho_{1}$ and $\rho_{2}$ are the densities of the SCS substrate and Al film, respectively. By substituting Eqs. (6) and (7) into Eq. (4), $E_{2}$ of the Al film can be obtained as

$$
E_{2}=E_{1}\left\{\left(\frac{f_{1+2}}{f_{1}}\right)^{2}\left(\frac{t_{1}}{t_{2}}+\frac{\rho_{2}}{\rho_{1}}\right)-\frac{t_{1}}{t_{2}}\right\}
$$

In this study, 2329 and $2700 \mathrm{~kg} / \mathrm{m}^{3}$ were applied to $\rho_{1}$ and $\rho_{2}$ for SCS and Al, respectively. ${ }^{(34,35)}$ Thus, by measuring $f_{1}$ and $t_{1}$ before the $\mathrm{Al}$ film deposition, and also by measuring $f_{1+2}$ and $t_{2}$ after the deposition, $E_{2}$ of the film can be obtained directly from the experiments.

\section{Experimental Setup}

Figure 3 shows a schematic of the experimental setup for the resonance test. The setup is composed of a manual prober (K-160MP, Kyowa Riken) with high-precision tungsten-carbide microprobes, a frequency response analyzer (FRA5022, NF Corporation), a high-speed bipolar amplifier (BA4825, NF Corporation), a digital microscope (VHX-1000, KEYENCE), and a computer. The probes were used to apply driving voltages ranging from 20 to $40 \mathrm{~V}_{\mathrm{pp}}$ with sinusoidal waveform to electrodes on device chips. During the device operation, the phase and amplitude signals from a capacitive sensor were recorded in each test. As shown in Fig. 4, the phase curves before and after the Al film deposition are found to be different. The frequency at the peak position in each phase curve denotes resonant frequency, which was determined by curve fitting with the Lorenz function. In this case, the resonant frequency of the SCS resonator

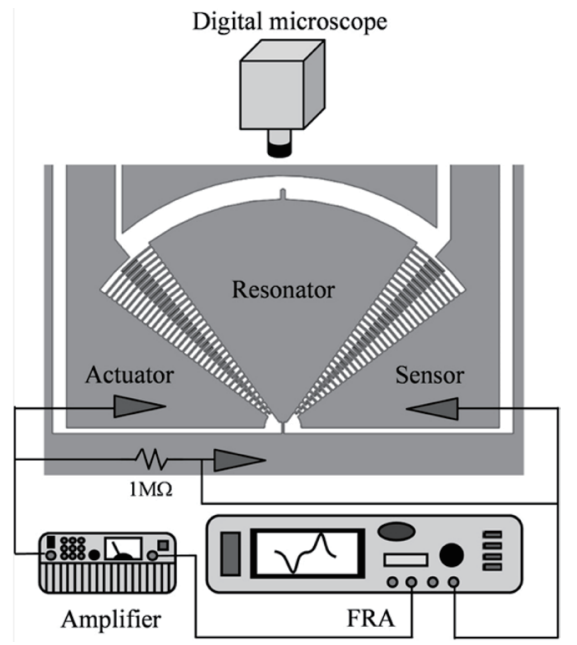

Fig. 3. Schematic of resonance test setup used in this work. 


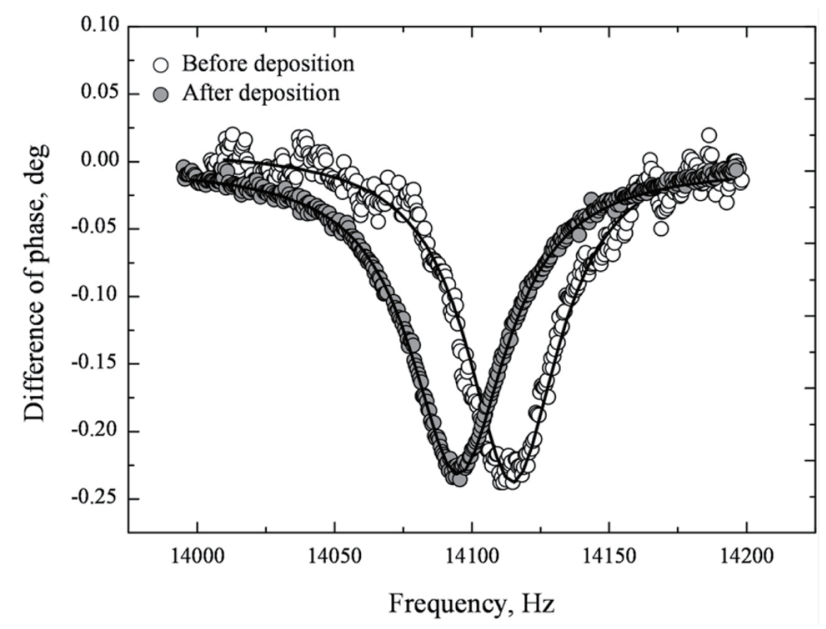

Fig. 4. Representative phase curves before and after Al film deposition. The frequencies at the peak positions of the two curves indicate the resonant frequencies of the resonator before and after Al film deposition. The difference is important in the derivation of Young's modulus of the film.

was $14113 \mathrm{~Hz}$. After the deposition of a 100-nm-thick Al film onto the resonator, the frequency was changed to $14098 \mathrm{~Hz}$. From these values, the film's Young's modulus can be calculated as $65.0 \mathrm{GPa}$ using Eq. (8). The resolution of the frequency measurement with the analyzer is $0.01 \mathrm{~Hz}$, and the accuracy of determining the resonant frequency is approximately $0.1 \mathrm{~Hz}$ at least. The thicknesses of the Al films were measured using an atomic force microscope (SPI4000, Seiko Instruments) with the resolution of $0.1 \mathrm{~nm}$ in the out-of-plane direction. The measurement accuracy is considered to be around $1 \mathrm{~nm}$. All the tests were carried out at ambient temperature in laboratory air.

\section{Experimental Results and Discussion}

\subsection{Resonance test results}

Figure 5 shows the relationship between the aspect ratio of the support beam and the difference in resonant frequencies before and after the $\mathrm{Al}$ film deposition. The aspect ratio $a$ was defined as the thickness-to-width ratio of the beam. The difference in resonant frequencies was calculated by subtracting the resonant frequency after the Al film deposition from that before the deposition. The circles and squares indicate the results obtained using resonators with beam aspect ratio larger and smaller than 1, respectively. The difference in the darkness of each plot color indicates the difference in the length of the support beam. That is, a darker colored plot denotes a result obtained using the resonator with a long support beam. The length $l$ of the beam ranges from 25 to $100 \mu \mathrm{m}$. The resonant frequencies of the resonators are found to definitely decrease after the Al film deposition owing to an increase in the whole mass. The difference in resonant frequencies decreases with the increase in the aspect ratio of the support beam as well as the increase in the beam length. This implies that a resonator with a 


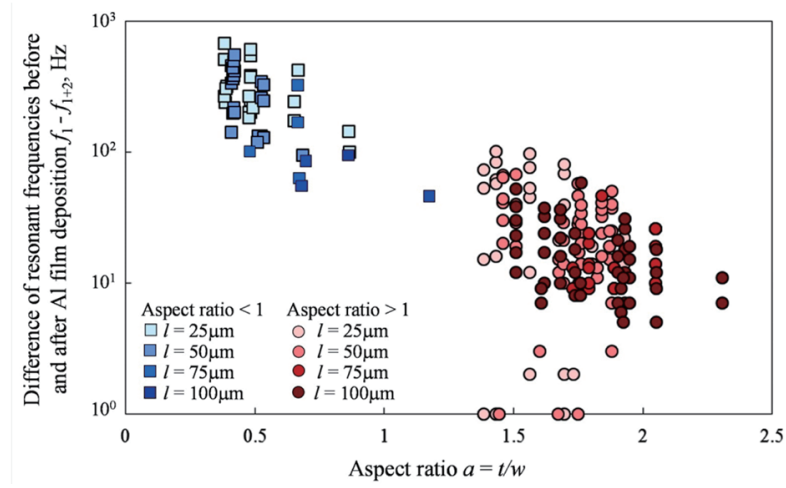

Fig. 5. (Color online) Relationship between aspect ratio of support beam and difference in resonant frequencies before and after Al film deposition.

low resonant frequency has a small difference in the frequencies between before and after the film deposition. The differences in resonant frequencies in each plot are scattered because the thicknesses of the evaporated $\mathrm{Al}$ films differ from each other. That is, the data of films of various thicknesses are included in each plot. The thicknesses of the films evaluated here range from 15 to $380 \mathrm{~nm}$.

Figure 6(a) shows the relationship between the aspect ratio of the support beam and Young's moduli of the Al films. The definition of the symbols used here is the same as that in Fig. 5. When the length and aspect ratio of the support beam are $25 \mu \mathrm{m}$ and 0.39 , respectively, Young's modulus of the $\mathrm{Al}$ film is $23.9 \mathrm{GPa}$ on average, which is $65.8 \%$ lower than the bulk value, $70 \mathrm{GPa}$. When the length increases to $50 \mu \mathrm{m}$ at almost the same aspect ratio, the mean Young's modulus also increases to $34.5 \mathrm{GPa}$. The trend that the moduli of Al films increase with increasing length and aspect ratio is the same as that for SCS. ${ }^{(21)}$ Young's modulus becomes close to the bulk value with increasing ratio. For example, when $l=100 \mu \mathrm{m}$ and $a=2.0$, the mean Young's modulus is $55.6 \mathrm{GPa}$, which is a $20.5 \%$ difference from the bulk value. Since the maximum deflection angle for all the resonators is kept constant at $4^{\circ}$ in each test, the resonators that have a longer support beam with a higher aspect ratio are more suitable for measuring Young's modulus from the viewpoint of the small strain theory. However, even with the same lengths and aspect ratios, the data of the moduli are found to be very scattered. In particular, several plots for $l=25 \mu \mathrm{m}$ and $a=1.2-1.8$ show that very high Young's moduli were obtained. The main reason for this is that the data for the Al films with various thicknesses ranging from 15 to $380 \mathrm{~nm}$ are included in each plot.

Figure 6(b) shows the relationship among Young's modulus of SCS, the structural material for resonators, and Young's moduli of Al films. When the SCS resonators have a short support beam and a small aspect ratio of the beam, the measured Young's moduli are definitely low, as previously reported. ${ }^{(21)}$ Young's moduli of Al films evaluated using the SCS resonators that can experimentally provide Young's moduli lower than $140 \mathrm{GPa}$ are also low, ranging from 19.7 to 47.2 GPa. This indicates that the resonators that cannot provide an SCS Young's modulus close to the ideal value (in this case, $168.9 \mathrm{GPa}$ for SCS (001)[110]) are not suitable for evaluating 


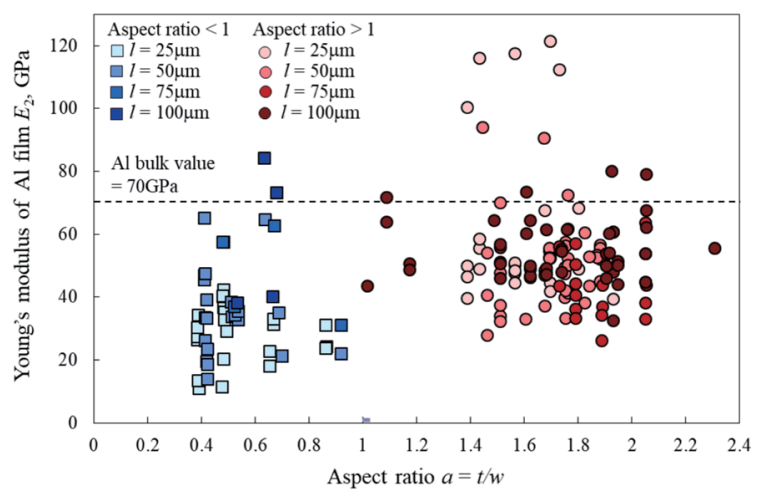

(a)

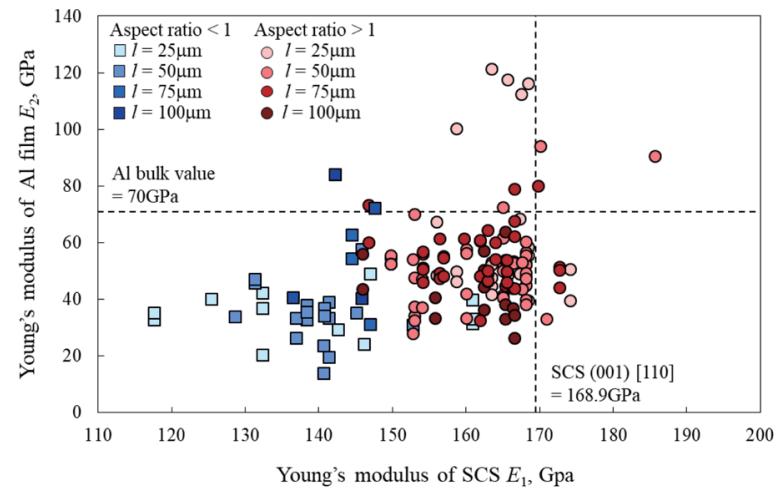

(b)

Fig. 6. (Color online) Measured Young's moduli by resonance testing: (a) aspect ratio of support beam vs measured Young's modulus of Al film and (b) relationship between measured Young's moduli of SCS and Al film.

Young's moduli of Al films. As the length and aspect ratio increase, Young's moduli of not only SCS but also Al films are found to increase toward 168.9 and $70 \mathrm{GPa}$, respectively, although data scatter for both materials is seen, as in Fig. 6(a). That is, this experimental finding means that, to accurately measure Young's moduli of submicron-thick films, the resonators that can be used to accurately measure Young's modulus of a structural material must be used in the tests.

As shown in Figs. 6(a) and 6(b), it was found that the measurement accuracy of Young's moduli of $\mathrm{Al}$ films is affected by the length and aspect ratio of the support beam. In addition, data scatter was observed; one reason for the data scatter in each plot may be the effect of film thickness. Figure 7 shows the effect of film thickness on Young's moduli of Al films. The squares and circles are indicative of Young's moduli obtained using the resonators with the support beam aspect ratio smaller and larger than 1.0, respectively. The pale-red circles indicate data obtained from all the resonators with $a \geq 1.0$. The dark-red plots indicate data from the resonators that can fulfill the conditions of $a \geq 1.0, l \geq 75 \mu \mathrm{m}$, and $E_{1} \geq 155 \mathrm{GPa}$. In the case of $a<1.0$, the mean Young's modulus of 260-nm-thick Al films is $38.1 \pm 5.5 \mathrm{GPa}$, which is almost half the bulk value. Even on decreasing the thickness to $90 \mathrm{~nm}$, the mean value was not changed significantly, whereas the standard deviation increased to $5.5 \mathrm{GPa}$. The mean Young's modulus throughout the thicknesses tested is $37.5 \pm 12.3 \mathrm{GPa}$. In all the data satisfying $a>1.0$, the mean Young's modulus appears almost constant at $51.9 \pm 8.8 \mathrm{GPa}$ in the thickness range from 50 to $380 \mathrm{~nm}$. When the thickness is $15 \mathrm{~nm}$, however, the mean Young's modulus abruptly increases to $101.0 \pm 13.0 \mathrm{GPa}$, which is an approximately $95.0 \%$ increase from the mean value of the films thicker than $50 \mathrm{~nm}$. The standard deviation also increases by $47.7 \%$ from the value for thicker films. For only the results under $a \geq 1.0, l \geq 75 \mu \mathrm{m}$, and $E_{1} \geq 155 \mathrm{GPa}$, the mean Young's modulus of the Al films thicker than $50 \mathrm{~nm}$ increases to $57.5 \pm 5.8 \mathrm{GPa}$, which is a $10.8 \%$ increase from the value at $a \geq 1.0$. The value becomes close to the ideal value, and the deviation is $17.9 \%$. This implies that, by using the resonators that enable accurate measurement of $E_{1}, E_{2}$ close to the true value can be measured. 


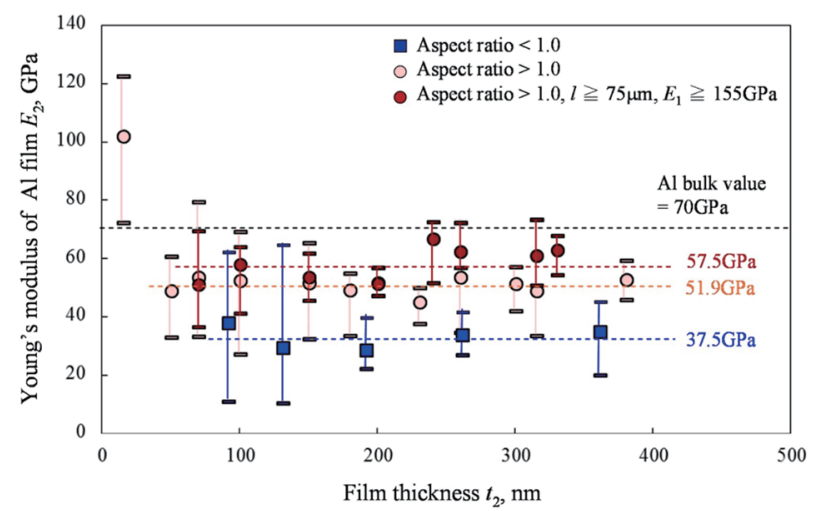

Fig. 7. (Color online) Relationship between Al film thickness and measured Young's modulus of the film. It is found that the aspect ratio of the support beam is very important in the derivation of Young's modulus of the Al film. No size dependences were found under any condition.

\subsection{Certainty of measured Young's modulus}

From the results in Fig. 7, Young's moduli of Al films thicker than $50 \mathrm{~nm}$ are considered to be unaffected by the thickness effect if the accuracies of the experiments for film thickness measurement and frequency measurement were sufficient for all the films tested. Before discussing the thickness effect, the effects of the cancellation of significant digits of measured film thickness and frequency on $E_{2}$ derivation are estimated and shown in Fig. 8. The solid and dashed lines indicate the estimation lines when the significant digits of 1.0 and $0.1 \mathrm{~Hz}$ for frequency measurement are accurate, respectively. The red and blue lines indicate the estimation lines when the significant digits of 1.0 and $0.1 \mathrm{~nm}$ for film thickness measurement are accurate, respectively. All the lines were calculated on the basis of the assumption that $57.5 \mathrm{GPa}$, experimentally obtained under the conditions of $a \geq 1.0, l \geq 75 \mu \mathrm{m}$, and $E_{1} \geq 155 \mathrm{GPa}$, is the true value for $E_{2}$. In addition to the data obtained under the above conditions, the data of $a \geq 1.0$ are also shown in the graph for comparison with the calculated lines. When the thickness is $250 \mathrm{~nm}$ at the accuracies of $1.0 \mathrm{~Hz}$ and $1.0 \mathrm{~nm}$, the simulated Young's moduli were 47.6 and $61.4 \mathrm{GPa}$, which deviate by -17.2 and $6.8 \%$, respectively, from the true value, $57.5 \mathrm{GPa}$, set here. At the same thickness, by improving the accuracy of film thickness measurement to $0.1 \mathrm{~nm}$, these estimated values were changed to 52.3 and $61.0 \mathrm{GPa}(-9.0$ and $6.1 \%$ deviations), respectively. By additional improvement of the frequency measurement accuracy to $0.1 \mathrm{~Hz}$, 56.3 and $58.2 \mathrm{GPa}$ (only -2.1 and $1.2 \%$ deviations) were estimated, respectively. Improving the frequency measurement accuracy by a single digit is found to be very effective for accurately measuring $E_{2}$. As the thickness decreases, the calculated lines gradually shift away from $57.5 \mathrm{GPa}$. At $100 \mathrm{~nm}$ thickness, the values under $1.0 \mathrm{~Hz}$ and $1.0 \mathrm{~nm}$ were calculated to be 38.1 and $69.5 \mathrm{GPa}$, which deviate by -33.7 and $20.9 \%$ from the true value, respectively. In the case of the same accuracies, the deviation abruptly increases with further decreasing thickness. For the same thickness, however, provided that the accuracy of frequency measurement is improved to $0.1 \mathrm{~Hz}$, the calculated values were 52.4 and $61.8 \mathrm{GPa}$, which deviate by only -8.9 and 


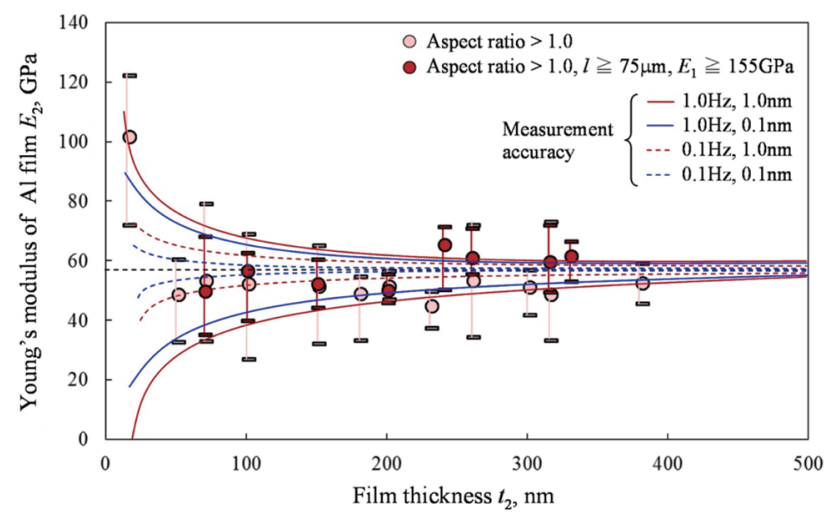

Fig. 8. (Color online) Effects of resonant frequency and film thickness measurement cancellations of significant digits on derivation of Young's modulus of Al film.

7.5\% from $57.5 \mathrm{GPa}$, respectively. Even at $50 \mathrm{~nm}$, the deviations were only -17.7 and $13.2 \%$, respectively, which will be allowable in nanoscale materials testing.

Since the resolutions of the frequency analyzer and atomic force microscope used for measuring resonant frequency and film thickness were $0.01 \mathrm{~Hz}$ and $0.1 \mathrm{~nm}$, respectively, these excellent measurement accuracies in the experiments can be estimated to be around $0.1 \mathrm{~Hz}$ and $1.0 \mathrm{~nm}$. In the films thicker than $50 \mathrm{~nm}$, Young's moduli obtained in the experiments were kept almost constant at around $55 \mathrm{GPa}$, although data scattering is seen in all the thickness ranges tested. The scatter might have been caused by not insufficient measurement accuracies but rather, the thickness nonuniformity of the films deposited on each resonator because the mean values at respective thicknesses were not so different. However, the mean Young's modulus of 15 -nm-thick Al films was $101.0 \mathrm{GPa}$, which is 1.8 times higher than the mean value for the films thicker than $50 \mathrm{~nm}$. There are many reports on the investigation of the effect of specimen size on the mechanical properties of Al thin films. ${ }^{(3,4,36-45)}$ The yield strength and fracture strength of sputtered Al films are well known to depend on the grain size $e^{(36,41,45)}$ and strain rate. $^{(42,43)}$ Moreover, several researchers have so far tried to conduct the nanoscale tensile testing of Al films with the size ranging from several tens to several hundreds of $\mathrm{nm}^{(3,4,36-40)}$ From their results, Young's modulus in the thickness range from 30 to $2000 \mathrm{~nm}$ seems not to deviate so much from the bulk value. For example, 30-nm-thick Al nanostructures showed 60.0 $\mathrm{GPa}$ and $2-\mu \mathrm{m}$-thick Al films showed $59.5 \mathrm{GPa} \cdot{ }^{(36,44)}$ Young's modulus of metals is well known to strongly depend on crystallographic texture, so that the difference in the deposition method might give rise to the difference in Young's modulus even if the specimen sizes are the same. Moreover, in the case of sputtered films, the diameters of columns in the vicinity of a substrate typically differ from the diameters close to the top surface. ${ }^{(46-48)}$ Since all the Al films evaluated in this paper were deposited by vacuum evaporation under the same conditions except for deposition time, it can be considered that these internal structures were the same. Therefore, considering the relationship between these obtained results and the measurement accuracies in the experiments, the obtained values at $15 \mathrm{~nm}$ are probably derived from not only the film thickness effect but also insufficient measurement accuracies plus the effect of a relatively 
large nonuniform thickness. In other words, this indicates that the resonance method with the experimental setup used here has the potential for the accurate measurement of Young's modulus of films thicker than $50 \mathrm{~nm}$ at least.

Figure 9 shows Young's modulus measured by resonance testing with that measured by nanoindentation testing. The pale- and dark-red circles are indicative of resonance test results using the resonators under the conditions of $a \geq 1.0$ and $a \geq 1.0$ plus $l \geq 75 \mu \mathrm{m}$ plus $E_{1} \geq 155 \mathrm{GPa}$, respectively. The triangles indicate nanoindentation test results. All the nanoindentation tests were carried out by the continuous stiffness measurement method to remove as completely as possible the effect of the substrate, which commonly appears in the tests for very thin films. ${ }^{(49,50)}$ In the films thicker than $100 \mathrm{~nm}$, the measured Young's moduli obtained from both the resonance and nanoindentation tests did not depend on the film thickness, although the mean values for each thickness were somewhat different from each other. The mean value, marked by the dark-red plots, is $57.5 \mathrm{GPa}$ in the resonance tests, which is comparable to the mean value of $61.4 \mathrm{GPa}$ in the nanoindentation tests. In the films thinner than $100 \mathrm{~nm}$, however, nanoindentation-derived Young's moduli are greatly scattered (for example, the mean values for thicknessess of 90 and $80 \mathrm{~nm}$ are 22.3 and $86.5 \mathrm{GPa}$, respectively), whereas resonance-derived Young's moduli do not change so much until the thickness decreases to less than $50 \mathrm{~nm}$. At around $20 \mathrm{~nm}$, the uncertainties of these two methods appear to be similar. These comparisons, therefore, reveal that the resonance test method is more advantageous for evaluating Young's modulus of sub-100-nm-thick thin films than the nanoindentation test method.

\subsection{Cause of lower Young's modulus of Al films}

The lower value of $58 \mathrm{GPa}$ measured in this study probably cannot be explained by crystallographic texture because the elastic anisotropy of $\mathrm{Al}$ is low $\left(E_{(111)} / E_{(100)}=1.19\right)$. Below, we discuss several possible factors behind the lower Young's modulus.

The first factor is the affected layer on the Si beam sidewalls produced by FIB milling. When a Si beam is fabricated by FIB with $40 \mathrm{kV}$, an approximately $25-40$-nm-thick Ga-

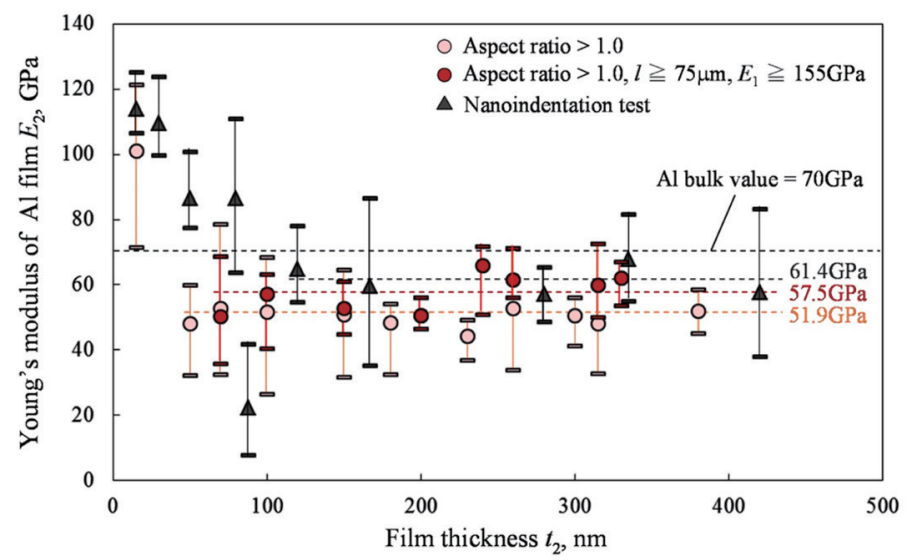

Fig. 9. (Color online) Young's modulus measured by resonance test and by nanoindentation test. 
included amorphous Si layer is sure to be produced on the sidewalls. ${ }^{(8)}$ Young's modulus of the affected layer can be estimated to be $80-110 \mathrm{GPa},{ }^{(7,8)}$ which is $35-53 \%$ lower than that of $\mathrm{Si}(001)[110]$. This indicates that it might be difficult to accurately provide Young's modulus of Si using the FIB-milled resonators, even though the sidewalls are flat. In this study, even in the resonators with the support beam length of $100 \mu \mathrm{m}$ and cross-sectional aspect ratio larger than 1.5, Young's modulus of Si ranged from 153 to $172 \mathrm{GPa}$. This means an approximately $9 \%$ deviation, at most, from the bulk value, $168.9 \mathrm{GPa}$. According to Eq. (8), since the resonators that showed a lower Young's modulus provide a lower Young's modulus of Al films, a lower measured value of $58 \mathrm{GPa}$ compared with the bulk value would have been obtained.

According to Eq. (8), film density may be one of the factors responsible for the lower Young's modulus. X-ray reflectometry analysis of the 100-nm-thick Al film prepared by vacuum evaporation was performed to directly measure the film density. The measured density of the film was $2704 \mathrm{~g} / \mathrm{cm}^{3}$, which was almost the same as the nominal value, $2700 \mathrm{~g} / \mathrm{cm}^{3}$. Thus, the effect of the density on the measured Young's modulus is considered to be small.

The effect of Poisson's ratio is considered to explain the lower measured value. The determination of Young's modulus in this work is based on the assumption that Poisson's ratio of the $\mathrm{Al}$ film is the same as that of the Si substrate, as represented in Eq. (6). If Poisson's ratio of the Al film differs from that of the Si substrate, using Eq. (5) is better. To quantitatively understand the difference between the two equations, the estimated Young's moduli of the Al film were compared. First, by inputting $70 \mathrm{GPa}$ for $E_{2}$ in Eq. (5), $E_{1+2}$ was calculated. Then, $E_{1+2}$ was input to Eq. (6); consequently, $E_{2}$ was estimated. The input and output values of $E_{2}$ were compared, as listed in Table 1. When the out-of-plane Poisson's ratio of Si was considered, the differences in $E_{2}$ between the two equations were within the range from 7.5 to $7.6 \%$. In the case of the in-plane Poisson's ratio, the differences were only approximately $0.1 \%$ owing to the closer value between these Poisson's ratios. Because of the strong anisotropy of $\mathrm{Si}$, the difference between Eqs. (5) and (6) for determining Young's modulus of the Al film can be estimated to be around $8 \%$ at most. For simple and easy derivation, we decided to use Eq. (6) to derive Young's modulus of Al films. The use of Eq. (5), which is better but highly complex, is a should be addressed in the future.

The surface irregularity of the deposited Al films is possibly related to the lower Young's modulus. A pure Al film is well known to show hillock formation on its surface. Although the photographs are not shown here, there are many hillocks with a diameter of less than 100 $\mathrm{nm}$ on all the $\mathrm{Al}$ films used in this work. The hillocks possibly cause stress relaxation of the

Table 1

Difference in $E_{2}$ between Eqs. (5) and (6). These differences show the Poisson's ratio effect on the derivation of Young's modulus.

\begin{tabular}{ccccccccc}
\hline $\begin{array}{c}t_{1} \\
(\mu \mathrm{m})\end{array}$ & $\begin{array}{c}t_{2} \\
(\mu \mathrm{m})\end{array}$ & \multicolumn{1}{c}{$v_{1}$} & $v_{2}$ & $\begin{array}{c}E_{1} \\
(\mathrm{GPa})\end{array}$ & $\begin{array}{c}E_{2} \text { in Eq. }(6) \\
(\mathrm{GPa})\end{array}$ & $\begin{array}{c}E_{2} \text { in Eq. }(5) \\
(\mathrm{GPa})\end{array}$ & $\begin{array}{c}E_{1+2} \\
(\mathrm{GPa})\end{array}$ & $\begin{array}{c}\text { Difference } \\
\text { in } E_{2} \text { between } \\
\text { Eqs. }(5) \text { and }(6)(\%)\end{array}$ \\
\hline 5 & 0.05 & 0.069 & 0.33 & 168.9 & 75.33 & 70 & 167.97 & 7.61 \\
5 & 0.1 & 0.069 & 0.33 & 168.9 & 75.30 & 70 & 167.06 & 7.57 \\
5 & 0.15 & 0.069 & 0.33 & 168.9 & 75.28 & 70 & 166.17 & 7.54 \\
5 & 0.2 & 0.069 & 0.33 & 168.9 & 75.25 & 70 & 165.30 & 7.51 \\
\hline
\end{tabular}


crystal structure, leading to low Young's modulus. Also, the hillocks definitely give rise to an increase in the uncertainty of the measured film thickness. Provided that the size and growth probability are independent of the film thickness, a thinner film would include a larger error than a thicker film in the measured Young's modulus. Even without hillocks, a slight surface undulation originating from crystal grains would give rise to the same tendency. Depositing a smoother and more uniform thin film is be necessary to effectively demonstrate the capability of the resonance method.

\section{Conclusions}

To demonstrate the effectiveness of the resonance test method for the direct measurement of Young's moduli of submicron-thick films, we investigated the effects of Al film thickness and SCS MEMS resonator geometry on the derivation of Young's modulus. After the film deposition, the resonant frequencies of the resonators having double layers of Al film and SCS substrate definitely decreased. The resonators that were able to provide Young's modulus close to the ideal value for SCS were also able to measure Young's modulus of the film close to the bulk value for Al. A large aspect ratio of the support beam of resonators was one of the key factors for accurately deriving Young's moduli of submicron-thick Al films as well as the SCS substrate. Young's moduli measured using appropriate resonators showed no thickness dependence for films thicker than $50 \mathrm{~nm}$. For films thinner than $50 \mathrm{~nm}$, owing to the accuracy limits in frequency and film thickness measurements, the obtained values abruptly increased. It was demonstrated that the resonance test method is superior to the nanoindentation test method in terms of applicable film thickness limit and data scatter.

\section{Acknowledgments}

The authors express their gratitude to Dr. S. Miyake and Mr. T. Kato of Kobelco Research Institute Inc. for performing the nanoindentation tests and for extensive discussion on this topic. This work was partly supported by a Grant-in-Aid of Hyogo COE Program Promotion Project.

\section{References}

1 Y. Nakai, Y. Kang, M. Okada, Y. Haruyama, K. Kanda, T. Ichihashi, and S. Matsui: Jpn. J. Appl. Phys. 49 (2010) 06GH07.

2 O. Tabata and T. Tsuchiya: Advanced Micro and Nanosystems (Wiley, New York, 2008) Vol. 6.

3 M. A. Haque and M. T. A. Saif: Exp. Mech. 43 (2003) 248.

4 M. A. Haque and M. T. A. Saif: Acta Mater. 51 (2003) 3053.

5 I. M. Graz, D. P. J. Cotton, and S. P. Lacour: Appl. Phys. Lett. 94 (2009) 071902.

6 T. Tsuchiya, Y. Ura, K. Sugano, and O. Tabata: J. Microelectromech. Syst. 21 (2012) 523.

7 T. Fujii, K. Sudoh, S. Sakakihara, M. Naito, S. Inoue, and T. Namazu: Jpn. J. Appl. Phys. 52 (2013) 110118.

8 T. Fujii, T. Namazu, K. Sudoh, S. Sakakihara, and S. Inoue: J. Eng. Mater. Technol. 135 (2013) 051002.

9 M. Yilmaz and J. W. Kysar: Nanotechnology 24 (2013) 165502.

10 H. J. Oh, H. Omori, M. Sadakata, I. Tsubokura, and Y. Isono: J. Microelectromech. Syst. 23 (2014) 994.

11 H. J. Oh, S. Kawase, I. Hanasaki, and Y. Isono: Jpn. J. Appl. Phys. 53 (2014) 027201.

12 B. Merle and M. Göken: Acta Mater. 59 (2011) 1772.

13 M. Berdova, T. Ylitalo, I. Kassamakov, J. Heino, P. T. Törmä, L. Kilpi, and S. Franssila: Acta Mater. 66 (2014) 370 . 
14 D. Passeri, A. Bettucci, M. Germano, M. Rossi, A. Alippi, A. Fiori, E. Tamburri, S. Orlanducci, M. L. Terranova, and J. J. Vlassak: Microelectron. Eng. 84 (2007) 490.

15 H. Ogi, N. Nakamura, and M. Hirao: Nondestr. Test. Eval. 26 (2011) 267.

16 A. Haim, S. Bar-Ad, and A. Azoulay: J. Phys. 278 (2011) 012005.

17 J. Wang, H. K. Kim, F. G. Shi, B. Zhao, and T. G. Nieh: Thin Solid Films 377 (2000) 413.

18 T. Namazu, N. Maruo, and S. Inoue: J. Mater. Sci. 47 (2012) 2725.

19 D. Goto, T. Namazu, T. Takeuchi, K. Murakami, E. Komatsu, Y. Kawashimo, and T. Takano: Proc. 15th Int. Conf. on Solid-State Sensors, Actuators and Microsystems, Transducers (2009) 1321-1324.

20 H. Yamagiwa, D. Goto, T. Namazu, T. Takeuchi, K. Murakami, Y. Kawashimo, T. Takano, K. Yoshiki, and S. Inoue: Extended Abstracts, Int. Conf. on Solid State Devices and Materials, SSDM (2010) 1212-1213.

21 S. Ito, H. Yamagiwa, T. Namazu, T. Takeuchi, K. Murakami, Y. Kawashimo, and T. Takano: Proc. 6th AsiaPacific Conf. on Transducers and Micro/Nano Technologies, APCOT (2012) ac12000070.

22 C. L. Muhlstein, S. B. Brown, and R. O. Ritchie: MRS Proc. 657 (2000) 5.

23 C. L. Muhlstein, S. B. Brown, and R. O. Ritchie: Sens. Actuators, A 94 (2001) 177.

24 C. L. Muhlstein, E. A. Stach, and R. O. Ritchie: Acta Mater. 50 (2002) 3579.

25 C. L. Muhlstein and R. O. Ritchie: Int. J. Fract. 120 (2003) 449.

26 C. L. Muhlstein, R. T. Howe, and R. O. Ritchie: Mech. Mater. 36 (2004) 13.

27 D. H. Alsem, E. A. Stach, C. L. Muhlstein, and R. O. Ritchie: Appl. Phys. Lett. 86 (2005) 041914.

28 C. L. Muhlstein, S. B. Brown, and R. O. Ritchie: J. Microelectromech. Syst. 10 (2001) 593.

29 O. N. Pierron and C. L. Muhlstein: J. Microelectromech. Syst. 15 (2006) 111.

30 R. O. Ritchie, J. J. Kruzic, C. L. Muhlstein, R. K. Nalla, and E. A. Stach: Int. J. Fract. 128 (2004) 1.

31 L. M. Zhang, D. Uttamchandani, B. Culshaw, and P. Dobson: Meas. Sci. Technol. 1 (1990) 1343.

32 K. K. Chawla: Composite Materials (Springer-Verlag, New York, 1998) p. 207.

33 Y. Isono, T. Namazu, and N. Terayama: J. Microelectromech Syst. 15 (2006) 169.

34 V. Lindroos, M. Tilli, A. Lehto, and T. Motooka: Handbook of Silicon Based MEMS Materials and Technologies (Elsevier, Amsterdam, 2009) p. 4.

35 W. M. Haynes: Handbook of Chemistry and Physics (CRC Press, Boca Raton, 1993) p. 130.

36 M. A. Haque and M. T. A. Saif: Scr. Mater. 47 (2002) 863.

37 M. A. Haque and M. T. A. Saif: Proc. Natl. Acad. Sci. U.S.A. 101 (2004) 6335.

38 H. D. Espinosa, B. C. Prorok, and B. Peng: J. Mech. Phys. Solids 52 (2004) 667.

39 D. S. Gianola, S. Van Petegem, M. Legros, S. Brandstetter, H. Van Swygenhoven, and K. J. Hemker: Acta Mater. 54 (2006) 2253.

40 D. T. Read, Y.-W. Cheng, R. R. Keller, and J. D. McColskey: Scr. Mater. 45 (2001) 583.

41 H. Huang and F. Spaepen: Acta Mater. 48 (2000) 3261.

42 E. Ben-David, T. Tepper-Faran, D. Rittel, and D. Shilo: Scr. Mater. 90-91 (2014) 6.

43 D. S. Gianola, D. H. Warner, J. F. Molinari, and K. J. Hemker: Scr. Mater. 55 (2006) 649.

44 C. T. Rosenmayer, F. R. Brotzen, and R. J. Gale: MRS Proc. 130 (1988) http://dx.doi.org/10.1557/PROC-13077.

45 D. Heinen, H. G. Bohn, and W. Schilling: J. Appl. Phys. 77 (1995) 3742.

46 J. W. Patten: Thin Solid Films 75 (1985) 205

47 J. W. Shin, J. Y. Lee, T. W. Kim, Y. S. No, W. J. Cho, and W. K. Choi: Appl. Phys. Lett. 88 (2006) 091911.

48 H. Jia, X. Wang, S. P. Pang, W. T. Zheng, B. H. Long, and B. Li: Trans. Nonferrous. Met. Soc. China 19 (2009) 353.

49 W. C. Oliver and G. M. Pharr: J. Mater. Res. 7 (1992) 1564.

50 W. C. Oliver and G. M. Pharr: J. Mater. Res. 19 (2004) 3. 